\title{
Alteraciones Hepáticas Causadas por el Sars-CoV-2
}

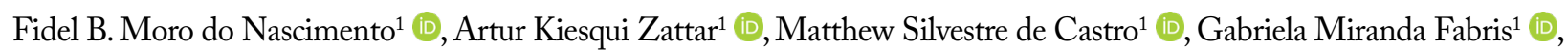
Vitória Carolina Bitencourt da Silva ${ }^{1}\left(\mathbb{D}\right.$, , Maria Eduarda Costa Oliveira $^{1}\left(\mathbb{D}\right.$, Lucas de Oliveira Rodrigues $^{1}\left(\mathbb{D}\right.$, Marina Zanatta $^{2}$

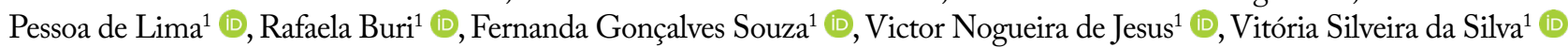

1. Centro Universitário de Várzea Grande Rớr Várzea Grande (MT), Brasil.

doi https://doi.org/10.53855/bjt.v25i1.427_esp

Autor correspondiente:

fidel.moro1@gmail.com

Editora de Sección

Ilka Boin

Recibido

Dez. 12, 2021

Aprobado

Jan. 27, 2022

Conflicto de interés

Nada que declarar.

\section{Cómo Citar}

Nascimento FBM, Zattar AK, Castro MS,

Fabris GM, Silva VCB, et al. Alteraciones

Hepáticas Causadas por el Sars-CoV-2.

BJT.2022; 25(01): e0622. https://doi. org/10.53855/bjt.v25i1.427_esp

eISSN

2764-1589

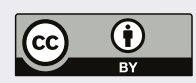

Resumen: Con la endemia del virus Sars-CoV-2, muchos individuos afectados por enfermedades hepáticas preexistentes como cirrosis hepática y las hepatopatías crónicas se tornaron expuestos a la descompensación del cuadro por la virulencia de la exposición y a la susceptibilidad individual por la infección del nuevo coronavirus. La citotoxicidad directa del virus Sars-CoV-2 ocurre por su replicación en las células hepáticas, dada por la conexión del agente a las célulasblanco por la expresión de la enzima convertidora de angiotensina 2 (ECA2), que es la principal mediadora de la replicación viral en los pacientes infectados por el Covid-19. Como consecuencia, las citoquinas proinflamatorias aumentan y pueden provocar hipoxia e isquemia sistémica. En asociación a la linfopenia y a la disminución de los niveles de células T-CD4+, los pacientes pueden evolucionar para descompensación o empeoramiento del cuadro infeccioso, con la insuficiencia hepática crónica agudizada desde la primera semana y, así, disminución de sobrevida. Los pacientes prediagnosticados con cirrosis e infectados por el virus del Covid-19 poseen mayor acometimiento hepático y peor pronóstico y, por tanto, merecen seguimiento especial, siendo cuidadosamente evaluados a fin de posibilitar la reducción de las lesiones hepáticas causadas por la infección.

Descriptores: Sars-CoV-2; Covid-19; Hepatocitos; Pandemias; Síndrome de la liberación de citoquina; Cirrosis hepática; Hipoxia.

\section{INTRODUCCIÓN}

El Covid-19 es una infección altamente contagiosa que surgió como endemia en diciembre del 2019 con sus primeros casos en la provincia china de Wuhan y enseguida evolucionó para una pandemia. ${ }^{1}$ La alta tasa de transmisibilidad por las vías interpersonal, contacto con superficies contaminadas y nosocomial es un factor que justifica su rápida diseminación mundial., ${ }^{2,3}$

Con el contagio por Sars-CoV-2, el virus ARN de cinta simple inicia la patogenicidad en el individuo. El proceso de invasión viral ocurre por medio de la conexión de gran afinidad de la proteína $S$ presente en el virus con la enzima convertidora de angiotensina 2 (ECA2), la cual está altamente presente en las vías aéreas. Esta conexión viabiliza la penetración en células-blanco alveolares por medio del proceso de endocitosis dependiente de clatrina, posibilitando así la fusión y la replicación viral dentro de las células alveolares y su propagación para otros segmentos del pulmón y demás sistemas que expresen ECA2. ${ }^{4,5}$ El acometimiento sistémico causado por la infección por Sars-CoV-2 fue observado en células cardíacas, renales, neurológicas y hepáticas, esta última verificada en diversos estudios mediante la alteración de exámenes de proteínas marcadoras de lesión hepática. ${ }^{6-12}$

El cuadro clínico de la infección viral es versátil, pudiendo ocurrir desde casos asintomáticos y sintomáticos clásicos, como fiebre, tos seca, fatiga, expectoración, disnea, 
dolor de garganta, cefalea, mialgia o artralgia, congestión nasal, diarrea, hemoptisis y congestión conjuntival. ${ }^{13}$ La gravedad de la infección varía de acuerdo con la virulencia de la exposición y la susceptibilidad del individuo afectado. La clasificación de factores de riesgo para casos de mayor gravedad del nuevo coronavirus es: edad avanzada, obesidad, diabetes mellitus, hipertensión arterial sistémica, enfermedades respiratorias crónicas, enfermedades cardiovasculares y neoplasias, evidenciando la importancia de las comorbilidades en los pacientes. ${ }^{14}$

La lesión hepática es un importante marcador de agravamiento de la infección en los individuos acometidos con la infección por el nuevo coronavirus, además de ser caracterizada como comorbilidad en pacientes que ya poseen enfermedades hepáticas preexistentes, como la cirrosis hepática descompensada y las hepatopatías crónicas. ${ }^{15-17}$ Esto se refleja directamente no solo en el daño causado en el hígado en el momento de la infección, sino también en el seguimiento a largo plazo de los pacientes, como demuestra el estudio APCOLIS. ${ }^{18}$ En este trabajo sobre la evolución de la enfermedad hepática preexistente en pacientes con infección por Sars-CoV-2, fue observada una aceleración de la lesión en el hígado en portadores de cirrosis cuando es comparado a individuos sin comorbilidades. ${ }^{18}$

Este estudio realiza una revisión no sistemática de los mecanismos de lesión hepática causados por el Sars-CoV-2 y su asociación con el prediagnóstico de cirrosis hepática, buscando analizar el agravamiento de la herida preexistente del hígado, posibles métodos de investigación de laboratorio y pronósticos de estos pacientes postinfección por el Covid-19.

\section{MECANISMOS DE LESIÓN HEPÁTICA POR SARS-COV-2}

Con la intención de comprender cómo el Covid-19 puede afectar al hígado, un estudio de cohorte fue realizado comparando diversos exámenes de laboratorio entre pacientes infectados que no presentasen complicaciones hepáticas. ${ }^{19}$ aunque el mecanismo de lesión hepática por el Covid-19 no haya sido completamente esclarecido, la investigación patológica de un paciente infectado demostró la presencia de esteatosis moderada, infiltración de leucocitos en las áreas lobulares y portal, necrosis focal y congestionamiento sinusoidal. ${ }^{19}$

\section{Citotoxicidad viral directa al hígado}

La citotoxicidad directa por causa de la replicación del virus en las células hepáticas se debe por la conexión del Sars-CoV-2 a las células-blanco, demostrada por la expresión de ECA2, siendo la principal mediadora de la replicación viral en los pacientes infectados por el Sars-CoV-2. ${ }^{20}$

Un estudio realizado por el Centro Universitario Médico de Groningen, en Holanda, analizó la distribución de ECA2 en los tejidos corporales, detectando la presencia de estas proteínas en los hepatocitos. ${ }^{21}$ Ya recientemente, en el 2020, hubo la publicación de un estudio de cohorte que reveló enriquecimiento significativo de la expresión de ECA2 en el hígado, principalmente en las células que componen los ductos biliares (colangiocitos), observando una media 20 veces mayor en relación al nivel de expresión en hepatocitos, mientras en las células de Kupffer la ECA2 no fue detectada. ${ }^{22}$ Reforzando esta propuesta, muestras de biopsia recolectadas de pacientes con Covid-19 evidencian esteatosis microvesicular moderada y actividad lobular y portal leve. ${ }^{23}$

Pacientes infectados por el virus durante la hospitalización en Wuhan demostraron aspartato aminotransferasa (AST), alanina aminotransferasa (ALT) y lactato deshidrogenasa (LDH) elevados, sugiriendo lesión relacionada a la progresión del Sars-CoV-2. ${ }^{24}$

\section{Lesión hepática inmunomediada por la respuesta sistémica del organismo}

Entre las posibles explicaciones para tales procesos está la lesión inmunomediada por la respuesta inflamatoria sistémica, conforme el relato de un estudio que evidenció las citoquinas posinflamatorias aumentadas principalmente en casos severos de Covid-19, lo que podría provocar hipoxia e isquemia sistémica. ${ }^{25}$

El aumento exacerbado de citoquinas inflamatorias fue descrito como tempestad de citoquinas, y, en conjunto, la linfopenia y la disminución de los niveles de células T-CD4+, hallazgos comunes en los pacientes infectados por Sars-CoV-2, pueden estar asociadas a la intensidad de la enfermedad y a la mortalidad. ${ }^{26}$

Uno de los estudios de cohorte caracterizó, en las citoquinas inflamatorias, la presencia de alteraciones cinéticas, incluyendo IL-6, IL-2, IL-4, IFN- $\gamma$ y TNF- $\alpha$ en el suero de los participantes. Con esto, se observó que en pacientes con características de infección leve por Sars-CoV-2 las fluctuaciones en los niveles séricos de esas citoquinas eran considerablemente menores que en pacientes graves, los cuales obtuvieron fluctuaciones significativas. ${ }^{27}$

Exceptuándose la IL-6, todas las otras citoquinas alcanzaron su pico en el suero entre tres y seis días después del comienzo de la enfermedad, no obstante los niveles de IL-6 y IL-10 demostraron un aumento mantenido en el grupo con un cuadro más grave si es comparado al otro con una infección más leve. En esta lógica, se vio que la disminución de las células T en el Covid-19 puede resultar en el agravamiento de las respuestas inflamatorias, mientras la normalización del número de estas células puede disminuir las respuestas inflamatorias. Reforzando tal hipótesis, el conteo de células $\mathrm{T}$ apuntaron para una correlación inversamente proporcional entre sus 
valores comparados a los niveles de citoquinas en la sangre periférica de pacientes graves, pues cuando las células T tenían los niveles más bajos ocurrió el pico en los niveles séricos de IL-10, IL-2, IL-4, TNF- $\alpha$ y IFN- $\gamma$, aproximadamente entre el cuarto y el sexto día. ${ }^{27}$

Ante las investigaciones, se observa un posible protagonismo de IL-6 en el proceso: el 30,39\% de los participantes con infección leve por Sars-CoV-2 tuvieron los valores de IL-6 más altos que lo normal, mientras en el grupo con infección severa el 76,19\% de los pacientes tuvieron su valor aumentado. A pesar de no ser completamente esclarecido, se considera posible que tal proceso ocurra en función de la inhibición de las células Th2 participantes de la inmunidad humoral en la etapa más inicial del Covid-19. ${ }^{28}$

\section{Efectos de la hipoxemia grave en el hígado}

Hay tres principales factores de riesgo que generan hipoxemia grave en los pacientes que evolucionan para acometimiento hepático: insuficiencia cardíaca, sepsis grave e insuficiencia respiratoria. ${ }^{29,30}$ En estas circunstancias, una tempestad de citoquinas perjudica el adecuado funcionamiento del hígado, dificultando la transducción para supervivencia celular, y provoca acumulación significativa de lípidos, como también el aumento del consumo de glicógeno y depleción de trifosfato de adenosina de los hepatocitos. Tales factores asociados causan estrés oxidativo y el aumento de los factores pro-inflamatorios. ${ }^{27,31,32}$

Como consecuencia, los pacientes evolucionan para un disturbio circulatorio derivado de una congestión pasiva y de la disminución de la perfusión hepática, el cual lleva a la hipoxia e isquemia. ${ }^{33}$ Con el daño hepático instalado, el edema intracelular de la organela estará presente. Para reducir tales efectos negativos, las células de Kupffer aumentan la producción de citoquinas para la activación de los leucocitos, lo que promueve un aumento de los niveles de transaminasa y lactato deshidrogenasa (LDH), generando la necesidad de soporte de oxígeno. ${ }^{3}$

La Fig. 1 demuestra la actuación simultánea de los mecanismos de lesión hepática con la invasión del Sars-Cov-2 en hepatocitos y colangiocitos, acarreando disfunciones irreversibles, con la consecuente muerte celular.

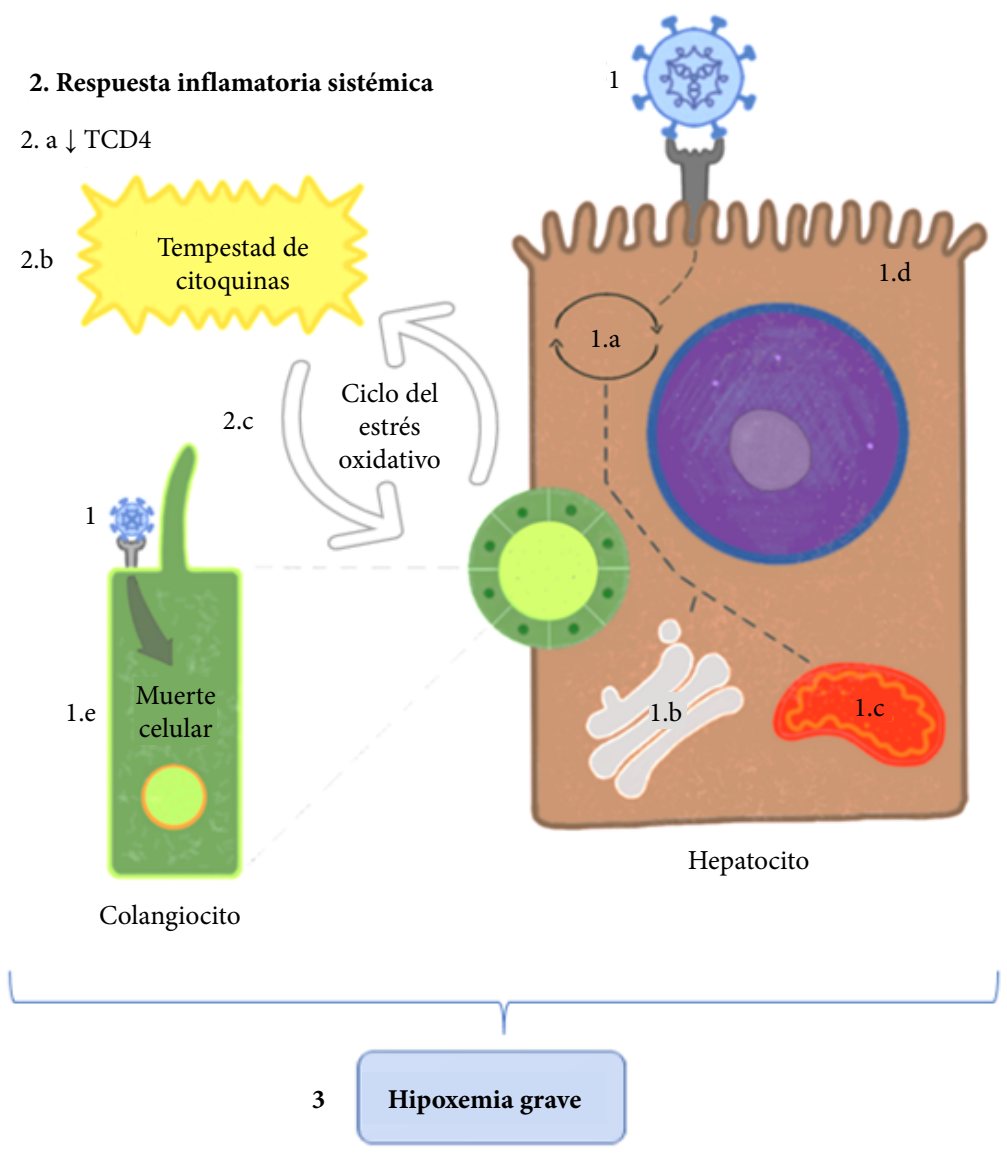

Figura 1. (1) El virus Sars-CoV-2 entra en la célula por medio del enlace con la enzima convertidora de angiotensina 2 (ECA2) expresa en la superficie de hepatocitos y colangiocitos. (1.a) Replicación viral. (1.b) Estrés del retículo endoplasmático. (1.c) Edema mitocondrial. (1.d) Lesión de membrana celular. (1.e) Muerte celular por causa de los daños irreversibles a las organelas. (2) Respuesta inflamatoria sistémica. (2.a) Linfopenia caracterizada por la disminución del conteo de linfocitos TCD4. (2.b) Aumento exacerbado de citoquinas inflamatorias. (2.c) Ciclo del estrés oxidativo: el ambiente proinflamatorio creado por las citoquinas dificulta el proceso de respiración celular, afectando, de este modo, la célula hepática. Como consecuencia, hay un nuevo aumento de los factores proinflamatorio.

(3) Hipoxemia grave, como resultado del disturbio circulatorio causado por el ambiente inflamatorio y células disfuncionales. 


\section{Evolución y pronóstico}

Pacientes en cuadros graves de Covid-19 sin comorbilidades hepáticas preexistentes demoran cerca de tres semanas para comenzar a desarrollar lesiones en el hígado. Sin embargo, pacientes previamente cirróticos en estado compensado que son afectados por la infección poseen acelerada progresión de lesión hepática, pudiendo ser perceptible la descompensación o la evolución de la herida con insuficiencia hepática crónica agudizada desde la primera semana. ${ }^{34,35}$

En estos pacientes es posible observar un aumento de citoquinas proinflamatorias, asociado con la presencia de marcadores inflamatorios de lesión hepática, ponderado por el aumento del nivel de las proteínas gama-glutamiltransferasa, ALT y AST en la corriente sanguínea. Estas proteínas fueron dosificadas como marcadores en estudios con pacientes cirróticos con Sars-CoV-2, además de la presencia de leucopenia, linfopenia y trombocitopenia en los exámenes de laboratorio. ${ }^{35-37}$

Como consecuencia del acometimiento hepático causado por el virus del Covid-19, el paciente cirrótico controlado puede evolucionar para un cuadro de descompensación. De esta forma, hay mayor daño al hígado del paciente y se disminuyen las oportunidades de sobrevida. ${ }^{38}$ Fue realizado un estudio basado en exámenes cadavéricos de pacientes que fallecieron por cirrosis descompensada y fue constatado que la invasión de células del hígado por el virus del Covid-19, asociada con edema mitocondrial y lesión de membrana celular, causa, por consiguiente, la apoptosis de hepatocitos, significativa inflamación focal lobular e infiltrado linfocito importante. ${ }^{39}$

La asociación de la cirrosis hepática con el nuevo coronavirus fue relacionada con posiciones superiores de gravedad hepática en la evaluación internacional representada por el sistema Child-Pugh, con 1/3 de los pacientes siendo reclasificados en categorías más severas. ${ }^{40}$ Los sistemas de evaluación del paciente hepatopata crónico son directamente proporcionales a la mortalidad por Sars-CoV-2. O sea, pacientes clasificados como Child-Pugh altos poseen mayores oportunidades de fallecer. ${ }^{41-43}$

De esta manera, es validado que pacientes prediagnosticados con cirrosis e infectados por el virus del Covid-19 poseen mayor acometimiento hepático y peor pronóstico..$^{37,38,40,41,43,46}$

\section{CONCLUSIÓN}

Hallazgos hepáticos son comunes en la infección por Covid-19, ocasionados por la citotoxicidad directa, por la lesión inmunomediada, por efectos hipoxémicos y, también, por el propio uso de medicamentos para tratamiento, sin embargo no hubo confirmación precisa con respecto a los efectos directos en la muerte de pacientes infectados por el Sars-CoV-2 demostrados en exámenes de autopsia. ${ }^{23}$ Pacientes con enfermedades hepáticas preexistentes merecen seguimiento especial, ya que las alteraciones clínicas del hígado se demuestran más intensas que lo normal, representando un peor pronóstico y aumento de la mortalidad en hepatopatas descompensados. ${ }^{40}$ Con base en las informaciones recolectadas, el manejo y el tratamiento del Covid-19 en pacientes deben ser cuidadosamente evaluados a fin de reducirse la lesión hepática causada por el Sars-CoV-2.

\section{CONTRIBUCIÓN DE LOS AUTORES}

Contribuciones científicas y intelectuales sustantivas al estudio: Nascimento FBM; Fabris GM; Souza FG; Castro MS; Oliveira MEC; Silva VS y Buri R; Concepción y diseño: Nascimento FBM; Fabris GM; Souza FG; Castro MS; Oliveira MEC; Silva VS y Buri R; Análisis y interpretación de datos: Silva VCB; Castro MS; Lima MKZ; Zattar AK; Jesus VN; Oliveira MEC; Silva VS; Rodrigues LO y Buri R; Escritura manuscrita: Nascimento FBM; Fabris GM; Silva VCB; Castro MS; Lima MKZ; Zattar AK; Jesus VN; Oliveira MEC; Silva VS; Rodrigues LO y Buri R; Aprobacíon final: Nascimento FBM; Castro MS; Zattar AK; Oliveira MEC; Silva VS y Buri R.

\section{DISPONIBILIDADE DE DADOS DE PESQUISA}

No se aplica.

\section{FINANCIAMENTO}

No se aplica. 


\section{AGRADECIMENTOS}

No se aplica.

\section{REFERENCIAS}

1. Zhu N, Zhang D, Wang W, Li X, Yang B, Song J, et al. A novel coronavirus from patients with pneumonia in China, 2019. New Eng J Med. 2020;382(8):727-33. https://doi.org/10.1056/NEJMoa2001017

2. Chan JFW, Yuan S, Kok KH, To KKW, Chu H, Yang J, et al. A familial cluster of pneumonia associated with the 2019 novel coronavirus indicating person-to-person transmission: a study of a family cluster. The Lancet. 2020;395(10223):514-23. https://doi.org/10.1016/s0140-6736(20)30154-9

3. Wang D, Hu B, Hu C, Zhu F, Liu X, Zhang J, et al. Clinical characteristics of 138 hospitalized patients with 2019 novel coronavirus-infected pneumonia in Wuhan, China. JAMA. 2020;323(11):1061-9. https://doi.org/10.1001/jama.2020.1585

4. Vaduganathan M, Vardeny P, Michel T, McMurray JJV, Pfeffer MA, Solomon SD. Renin-angiotensin-aldosterone system inhibitors in patients with Covid-19. N Engl J Med. 2020;382(17):1653-9. https://doi.org/10.1056/nejmsr2005760

5. Ye M, Wysocki J, William J, Soler MJ, Cokic I, Batlle D. Glomerular localization and expression of angiotensin-converting enzyme 2 and angiotensin-converting enzyme: Implications for albuminuria in diabetes. J Am Soc Nephrol. 2006;17(11):306775. https://doi.org/10.1681/asn.2006050423

6. Dweck MR, Bularga A, Hahn RT, Bing R, Lee KK, Chapman AR, et al. Global evaluation of echocardiography in patients with COVID-19. Eur Heart J Cardiovasc Imaging. 2020;21(9):949-58. https://doi.org/10.1093/ehjci/jeaa178

7. Cheng Y, Luo R, Wang K, Zhang M, Wang Z, Dong L, et al. Kidney disease is associated with in-hospital death of patients with COVID-19. Kidney Int. 2020;97(5):829-38. https://doi.org/10.1016/j.kint.2020.03.005

8. Mao L, Jin H, Wang M, Hu Y, Chen S, He Q, et al. Neurologic manifestations of hospitalized patients with coronavirus disease 2019 in Wuhan, China. JAMA Neurol. 2020;77(6):683-90. https://doi.org/10.1001/jamaneurol.2020.1127

9. Bernal-Monterde V, Casas-Deza D, Letona-Giménez L, Llama-Celis N de la, Calmarza P, Sierra-Gabarda O, et al. SARS-CoV-2 infection induces a dual response in liver function tests: Association with mortality during hospitalization. Biomedicines. 2020;8(9):328. https://doi.org/10.3390/biomedicines8090328

10. Guan W, Ni Z, Hu Y, Liang W, Ou C, He J, et al. Clinical characteristics of coronavirus disease 2019 in China. New Engl J Med. 2020;382(18):1708-20. https://doi.org/10.1056/NEJMoa2002032

11. Chen N, Zhou M, Dong X, Qu J, Gong F, Han Y, et al. Epidemiological and clinical characteristics of 99 cases of 2019 novel coronavirus pneumonia in Wuhan, China: a descriptive study. The Lancet. 2020;395(10223):507-13. https://doi.org/10.1016/s0140-6736(20)30211-7

12. Cai Q, Huang D, Yu H, Zhu Z, Xia Z, Su Y, et al. COVID-19: Abnormal liver function tests. J Hepatol. 2020;73(3):566-74. https://doi.org/10.1016/j.jhep.2020.04.006

13. World Health Organization. Mission China Joint. Report of the WHO-China Joint Mission on coronavirus disease 2019 (COVID-19). The WHO-China Joint Mission on Coronavirus Disease 2019. World Health Organization; 2020.

14. Galvão MHR, Roncalli AG. Factors associated with increased risk of death from covid-19: A survival analysis based on confirmed cases. Rev Bras Epidemiol. 2021;23:e200106. https://doi.org/10.1590/1980-549720200106

15. Bellentani S, Saccoccio G, Costa G, Tiribelli C, Manenti F, Sodde M, et al. Drinking habits as cofactors of risk for alcohol induced liver damage. Gut. 1997;41(6):845-50. https://doi.org/10.1136/gut.41.6.845

16. Poynard T, Bedossa P, Opolon P. Natural history of liver fibrosis progression in patients with chronic hepatitis C. The Lancet. 1997;349(9055):825-32. https://doi.org/10.1016/s0140-6736(96)07642-8

17. Farrell GC, Larter CZ. Nonalcoholic fatty liver disease: from steatosis to cirrhosis. Hepatology. 2006;43(2 Suppl. 1):S99-S112. https://doi.org/10.1002/hep.20973

18. Sarin SK, Choudhury A, Lau GK, Zheng MH, Ji D, Abd-Elsalam S, et al. Pre-existing liver disease is associated with poor outcome in patients with SARS CoV2 infection; The APCOLIS Study (APASL COVID-19 Liver Injury Spectrum Study). Hepatology Int. 2020;14(5):690-700. https://doi.org/10.1007/s12072-020-10072-8

19. Lei P, Zhang L, Han P, Zheng C, Tong Q, Shang H, et al. Liver injury in patients with COVID-19: clinical profiles, CT findings, the correlation of the severity with liver injury. Hepatol Int. 2020;14(5):733-42. https://doi.org/10.1007/s12072-020-10087-1

20. Li W, Moore MJ, Vasilieva N, Sui J, Wong SK, Berne MA, et al. Angiotensin-converting enzyme 2 is a functional receptor for the SARS coronavirus. Nature. 2003;426(6965):450-4. https://doi.org/10.1038/nature02145

21. Hamming I, Timens W, Bulthuis MLC, Lely AT, Navis GJ, van Goor H. Tissue distribution of ACE2 protein, the functional receptor for SARS coronavirus. A first step in understanding SARS pathogenesis. J Pathol. 2004;203(2):631-7. https://doi. org/10.1002/path.1570 
22. Chai X, Hu L, Zhang Y, Han W, Lu Z, Ke A, et al. Specific ACE2 expression in cholangiocytes may cause liver damage after 2019-nCoV infection. BioRxiv. 2020. https://doi.org/10.1101/2020.02.03.931766

23. Xu Z, Shi L, Wang Y, Zhang J, Huang L, Zhang C, et al. Pathological findings of COVID-19 associated with acute respiratory distress syndrome. Lancet Respir Med. 2020;8(4):420-2. https://doi.org/10.1016/s2213-2600(20)30076-X

24. Wang D, Hu B, Hu C, Zhu F, Liu X, Zhang J, et al. Clinical characteristics of 138 hospitalized patients with 2019 novel coronavirus-infected pneumonia in Wuhan, China. JAMA. 2020;323(11):1061-9. https://doi.org/10.1001/jama.2020.1585

25. Wang Y, Liu S, Liu H, Li W, Lin F, Jiang L, et al. SARS-CoV-2 infection of the liver directly contributes to hepatic impairment in patients with COVID-19. J Hepatol. 2020;73(4):807-16. https://doi.org/10.1016\%2Fj.jhep.2020.05.002

26. Zhang C, Shi L, Wang FS. Liver injury in COVID-19: management and challenges. Lancet Gastroenterol Hepatol. 2020;5(5):428-30. https://doi.org/10.1016/s2468-1253(20)30057-1

27. Liu J, Li S, Liu J, Liang B, Wang X, Wang H, et al. Longitudinal characteristics of lymphocyte responses and cytokine profiles in the peripheral blood of SARS-CoV-2 infected patients. Ebiomedicine. 2020;55:102763. https://doi.org/10.1016/j.ebiom.2020.102763

28. Wan S, Yi Q, Fan S, Lv J, Zhang X, Guo L, et al. Characteristics of lymphocyte subsets and cytokines in peripheral blood of 123 hospitalized patients with2019novel coronaviruspneumonia(NCP).Medrxiv.https://doi.org/10.1101/2020.02.10.20021832

29. Birrer R, Takuda Y, Takara T. Hypoxic hepatopathy: pathophysiology and prognosis. Intern Med. 2007;46(14):1063-70. https://doi.org/10.2169/internalmedicine.46.0059

30. Wong CK, Lam CWK, Wu AKL, Ip WK, Lee NLS, Chan IHS, et al. Plasma inflammatory cytokines and chemokines in severe acute respiratory syndrome. Clin Exp Immunol. 2004;136(1):95-103. https://doi.org/10.1111/j.1365-2249.2004.02415.x

31. Kulkarni A, Kumar P, Tevethia HV, Premkumar M, Arab JP, Candia R, et al. Systematic review with meta-analysis: liver manifestations and outcomes in COVID-19. Aliment Pharmacol Ther. 2020;52(4):584-99. https://doi.org/10.1111/apt.15916

32. Bloom PP, Meyerowitz EA, Reinus Z, Daidone M, Gustafson J, Kim AY, et al. Liver Biochemistries in Hospitalized Patients With COVID-19. Hepatology. 2021;73(3):890-900. https://doi.org/10.1002/hep.31326

33. Varga Z, Flammer AJ, Steiger P, Haberecker M, Andermatt R, Zinkernagel AS, et al. Endothelial cell infection and endotheliitis in COVID-19. Lancet. 2020;395(10234):1417-8. https://doi.org/10.1016/s0140-6736(20)30937-5

34. Sarin SK, Choudhury A, Lau GK, Zheng M-H, Ji D, Abd-Elsalam S, et al. Pre-existing liver disease is associated with poor outcome in patients with SARS CoV2 infection; The APCOLIS Study (APASL COVID-19 Liver Injury Spectrum Study). Hepatol Int. 2020;14(5):690-700. https://doi.org/10.1007/s12072-020-10072-8

35. Ji D, Zhang D, Yang T, Mu J, Zhao P, Xu J, et al. Effect of COVID-19 on patients with compensated chronic liver diseases. Hepatol Int. 2020;14(5):701-10. https://doi.org/10.1007/s12072-020-10058-6

36. Marjani A, Mehrpouya M, Pourhashem Z. Serum $\gamma$-glutamyltransferase, alanine aminotransferase and aspartate aminotransferase activity in healthy blood donor of different ethnic groups in Gorgan. J Clin Diagn Res. 2016;10(7):BC04-6. https://doi.org/10.7860\%2FJCDR\%2F2016\%2F20919.8181

37. Melo APS, França EB, Malta DC, Garcia LP, Mooney M, Naghavi M. Mortalidade por cirrose, câncer hepático e transtornos devidos ao uso de álcool: Carga Global de Doenças no Brasil, 1990 e 2015. Rev Bras Epidemiol. 2017;20(Supl. 1):61-74. https://doi.org/10.1590/1980-5497201700050006

38. Kim D, Adeniji N, Latt N, Kumar S, Bloom PP, Aby ES, et al. Predictors of outcomes of COVID-19 in patients with chronic liver disease: US multi-center study. Clin Gastroenterol Hepatol. 2021;19(7):1469-79. https://doi.org/10.1016/j.cgh.2020.09.027

39. Rela M, Patil V, Narasimhan G, Jothimani D. COVID-19 in decompensated cirrhosis. Hepatol Int. 2020;14(6):1125-7. https:// doi.org/10.1007/s12072-020-10092-4

40. Qi X, Liu Y, Wang J, Fallowfield JA, Wang J, Li X, et al. Clinical course and risk factors for mortality of COVID-19 patients with pre-existing cirrhosis: A multicentre cohort study. Gut. 2021;70(2):433-6. https://doi.org/10.1136/gutjnl-2020-321666

41. Iavarone M, D’Ambrosio R, Soria A, Triolo M, Pugliese N, del Poggio P, et al. High rates of 30-day mortality in patients with cirrhosis and COVID-19. J Hepatol. 2020;73(5):1063-71. https://doi.org/10.1016/j.jhep.2020.06.001

42. Marjot T, Moon AM, Cook JA, Abd-Elsalam S, Aloman C, Armstrong MJ, et al. Outcomes following SARS-CoV-2 infection in patients with chronic liver disease: An international registry study. J Hepatol. 2021;74(3):567-77. https://doi.org/10.1016/j. jhep.2020.09.024

43. Qi X, Wang J, Li X, Wang Z, Liu Y, Yang H, et al. Clinical course of COVID-19 in patients with pre-existing decompensated cirrhosis: initial report from China. Hepatol Int. 2020;14(4):478-82. https://doi.org/10.1007/s12072-020-10051-z

44. Shalimar, Elhence A, Vaishnav M, Kumar R, Pathak P, Soni KD, et al. Poor outcomes in patients with cirrhosis and corona virus disease-19. Indian J Gastroenterol. 2020;39(3):285-91. https://doi.org/10.1007/s12664-020-01074-3

45. Bajaj JS, Garcia-Tsao G, Biggins SW, Kamath PS, Wong F, McGeorge S, et al. Comparison of mortality risk in patients with cirrhosis and COVID-19 compared with patients with cirrhosis alone and COVID-19 alone: multicentre matched cohort. Gut. 2021;70(3):531-6.

46. Hashemi N, Viveiros K, Redd WD, Zhou JC, McCarty TR, Bazarbashi AN, et al. Impact of chronic liver disease on outcomes of hospitalized patients with COVID-19: A multicentre United States experience. Liver Int. 2020;40(10):2515-21. https://doi. org/10.1111/liv.14583 